\title{
MRI determination of the vertebral termination of the dural sac tip in a South African population: clinical significance during spinal irradiation and caudal anaesthesia
}

\author{
A Cilliers, $M B$ ChB, DA(SA) \\ D H Schulenburg, MMedSc, MB ChB \\ J Janse van Rensburg, MB ChB, MMed (Diagn \\ Rad), Dip Gen Intervent Radiol \\ Department of Diagnostic Radiology, Faculty of Health Sciences, University \\ of the Free State, Bloemfontein
}

\begin{abstract}
Knowledge of the approximate caudal termination of the dural sac (DS) is important, especially when placing the portal fields during lower craniospinal irradiation (CSI) and performing caudal anaesthesia. The purpose of this investigation was to determine the level of termination of the DS in relation to the spine in a group of South African patients by using magnetic resonance imaging (MRI). We retrospectively reviewed the lumbosacral MR imaging of 309 patients, in each case identifying the tip of the DS. This level was recorded in relation to the adjacent lumbosacral vertebral body i.e. upper-, middle- and lower-third and adjacent intervertebral disc. The overall mean of the DS position was at the middle third of S2. Although the caudal DS tip was at the level of S2 in the majority of patients, a notable percentage (13.9\%) had a DS tip level lower than the lower third of S2, and 15.2\% had levels higher than the S1-S2 intervertebral disc. This study failed to demonstrate a difference in the DS termination level, compared with the levels reported in various international studies; nor is there a statistical difference between gender, race and age. Our study shows that routine placement of the portal field at the lower border of S2 adequately treats the majority of CSI patients. However, some patients (13.9\%) will be undertreated and some patients (15.2\%) will be overradiated. Using spinal MRI to establish the lower border of the CSI portal field will, however, benefit patients by ensuring adequate coverage of the entire neuroaxis as well as minimising late gonadal toxicity owing to overradiation.
\end{abstract}

\section{Introduction}

The spinal cord ends at the level of the L1-L2 disk space, with the nerve roots extending caudally in an enclosed sac known as the dural sac (DS). The DS protects the dangling nerve roots and is made up of two distinct but tightly bound layers called the dura mater and arachnoid mater.

Generally, the mean level of termination of the DS, which has been described in the standard textbook and some cadaveric studies, is located at the second sacral vertebral level. ${ }^{1-3}$ However, cadaveric dissection is subject to distortion, and measurements may alter after skeletonisation of the cadaver. ${ }^{4}$ Variations in the level of the DS termination were shown in previous studies where the DS extended caudally to the S2 level. ${ }^{3,5}$

In defining and/or placing the distal portal fields for CSI in the treatment of cerebrospinal fluid (CSF) seeding or leptomeningeal metastasis, the radiotherapist needs to know the level of the DS ending to ensure complete coverage of the entire subarachnoid space. In patients where the DS tip ends very low, the distal part of the spinal column might be excluded from the radiation field, whereas patients with a DS ending higher than expected will receive unnecessary irradiation to their pelvic organs.

A caudal approach to the extradural space (caudal block) is used for intra- and postoperative analgesia in a variety of operations, as well as the management of chronic pain. A detailed understanding of the anatomy of the caudal region of the extradural space is therefore desirable for clinicians using this technique. The DS ending is most relevant to clinical practice as the length of the needle used to perform the caudal block should be carefully chosen to avoid puncturing the dura. ${ }^{6}$

The development of MRI has greatly enhanced our understanding of the living human anatomy. With the help of MRI, it is possible to determine the vertebral level of termination of the DS in the living individual. ${ }^{7}$ The level of the caudal DS tip in the adult population, as determined by MRI, has been described in various studies. ${ }^{5,6,89}$ These studies were, however, all limited in the number of cases reviewed. Knowing the caudal termination of the DS in the South African population is important, especially for more precise performance of the distal spinal part of a CSI, and also for caudal anaesthetic blocks.

The purpose of this investigation was to determine by using MRI the level of termination of the DS in relation to the spine in a group of South African patients, and to compare this position among age groups, gender, and black and white racial groups.

\section{Material and methods}

T1 and T2 sagittal spin-echo MR images made between October 2007 and November 2008 of the lumbar-sacral spine of 526 patients were retrospectively reviewed in the Department of Diagnostic Radiology, Universitas Hospital, Bloemfontein. Of this number, 217 (41.3\%) of these examinations were excluded from our study owing to previous spinal surgery, presence of congenital anomalies such as scoliosis, severe degenerative changes and malignancies in the lumbosacral area, and in patients where identification of the DS was difficult. Data from 309 (58.7\%) patient records were included in the statistical analysis.

The study group consisted of patients who were referred to the MR scanner for assessment of possible causes of lower back pain and 


\section{ORIGINAL ARTICLE}

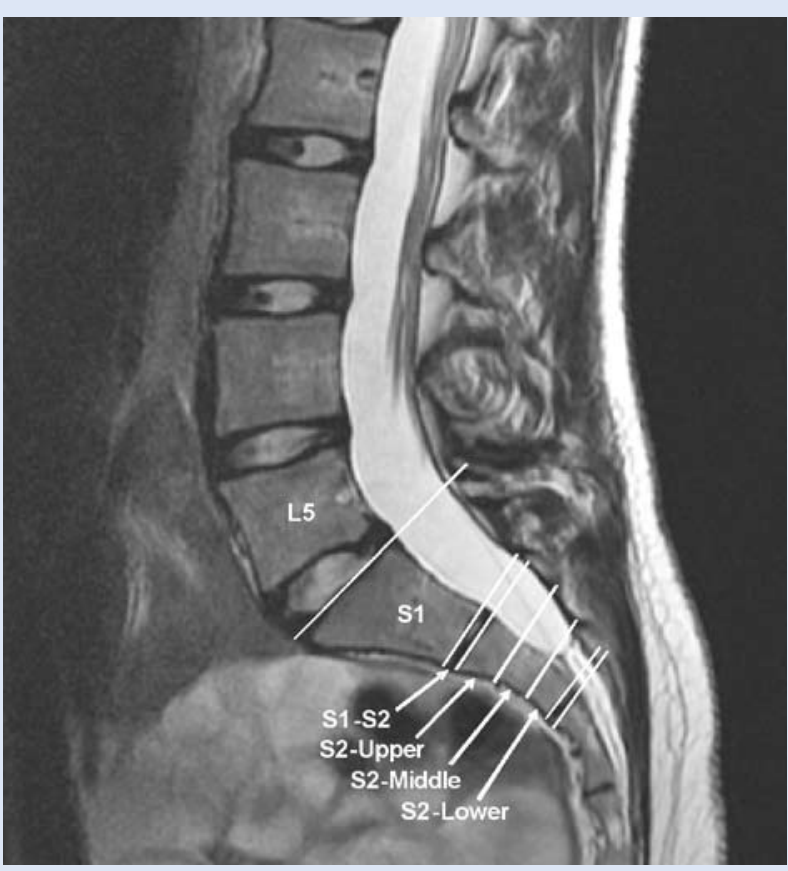

Fig. 1. A midline, sagittal, T2-weighted, spin-echo MR image of the lumbosacral spine demonstrating our method for determining the position of the tip of the dural sac.

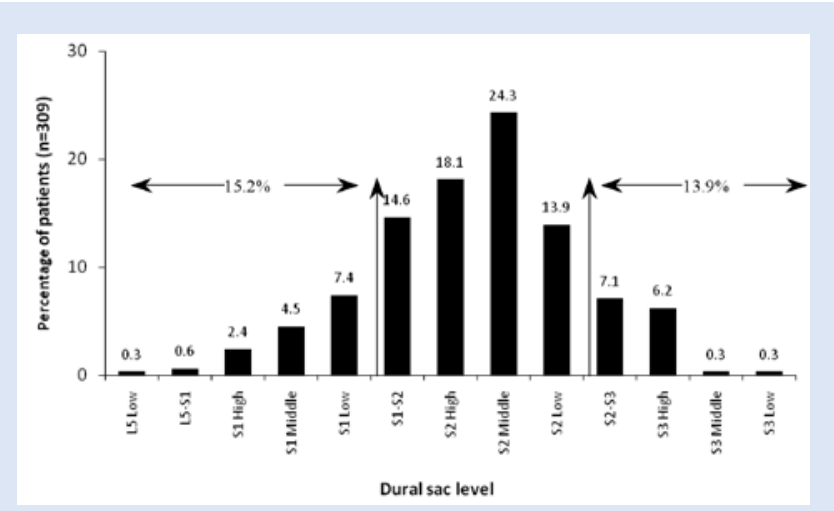

Fig. 2. Distribution of different levels of the dural sac tip as determined by means of a T2-weighted MRI of the lumbar spine.

radiculopathy. MR scans were reported as either normal or with minor age-related degenerative changes. Fig. 1 shows an MR image of the lumbosacral spine to demonstrate our method for determining the position of the caudal tip of the DS.

MRI of the spine was performed on a Signa 1.5T (GE Medical Systems, Milwaukee, USA) using a phased array spine coil, with the patient supine. Sagittal images were acquired using a slice thickness of $4.0 \mathrm{~mm}$ and a $1 \mathrm{~mm}$ interslice gap. Although both T1- and T2-weighted sagittal images were available, the relevant anatomy was better demonstrated by the latter imaging protocol.

Each patient's age, sex, race and location of the lower limit of the DS were recorded. The most caudal level of the DS was determined in relation to the adjacent vertebra and disc space. This was done by

\begin{tabular}{|lrrr|}
\hline \multicolumn{4}{|c}{ Table I. Comparison of DS tip levels by race } \\
\hline & \multicolumn{2}{c}{ No. of patients $(\%)$} & \\
\cline { 2 - 3 } Level of DS tip & \multicolumn{1}{c}{ Black } & White & \\
L5 low & $0(0)$ & $1(0.5)$ & 1.000 \\
L5-S1 disc space & $1(0.9)$ & $1(0.5)$ & 1.000 \\
S1 high & $2(1.7)$ & $5(2.6)$ & 0.712 \\
S1 middle & $6(5.1)$ & $8(4.2)$ & 0.781 \\
S1 low & $11(9.3)$ & $12(6.3)$ & 0.323 \\
S1-S2 disc space & $19(16.1)$ & $26(13.6)$ & 0.547 \\
S2 high & $20(16.9)$ & $36(18.8)$ & 0.674 \\
S2 middle & $27(22.9)$ & $48(25.1)$ & 0.654 \\
S2 low & $13(11.0)$ & $30(15.7)$ & 0.247 \\
S2-S3 disc space & $13(11.0)$ & $9(4.7)$ & 0.036 \\
S3 high & $5(4.2)$ & $14(7.3)$ & 0.272 \\
S3 middle & $0(0)$ & $1(0.5)$ & 1.000 \\
S3 low & $1(0.9)$ & $0(0)$ & 0.382 \\
& & & \\
\hline
\end{tabular}

\begin{tabular}{|lrrr|}
\hline \multicolumn{4}{|c}{ Table II. Comparison of DS tip levels by gender } \\
\hline \multicolumn{4}{c}{ No. of patients (\%) } \\
\cline { 2 - 3 } Level of DS tip & \multicolumn{1}{c}{ Male } & \multicolumn{1}{c|}{ Female } & \\
L5 low & $1(0.8)$ & $(\boldsymbol{N}=\mathbf{1 7 6})$ & p-value \\
L5-S1 disc space & $0(0)$ & $2(1.1)$ & 0.5081 \\
S1 high & $1(0.8)$ & $6(3.4)$ & 0.2456 \\
S1 middle & $8(6.0)$ & $6(3.4)$ & 0.2856 \\
S1 low & $11(8.3)$ & $12(6.8)$ & 0.6659 \\
S1-S2 disc space & $23(17.3)$ & $22(12.5)$ & 0.2750 \\
S2 high & $19(14.3)$ & $37(21.0)$ & 0.1383 \\
S2 middle & $34(25.6)$ & $41(23.3)$ & 0.6885 \\
S2 low & $16(12.0)$ & $27(15.3)$ & 0.5071 \\
S2-S3 disc space & $10(7.5)$ & $12(6.8)$ & 0.8266 \\
S3 high & $9(6.8)$ & $10(5.7)$ & 0.8120 \\
S3 middle & $0(0)$ & $1(0.6)$ & 1.0000 \\
S3 low & $1(0.8)$ & $0(0)$ & 0.4304 \\
& & & \\
\hline
\end{tabular}

extending a line, perpendicular to the long axis of the dura, across to the adjacent vertebrae and disc space. In accordance with the method of Saifuddin et $a l,{ }^{10}$ each vertebra was divided into 3 equal portions (upper, middle and lower thirds respectively) and the intervertebral disc was defined as a separate region (see Fig. 1.) The image that showed the most inferior convergence point of the DS was used for measurement. For statistical evaluation, each lumbosacral vertebral regional level of the caudal DS tip was assigned a number; for example, the upper third of L5 $=1$ and the upper third of S $4=17$.

Results were summarised by the Department of Biostatistics according to frequencies and percentages (categorical variables) and means, standard deviation or percentiles (numerical variables). Subgroups (race, gender and age groups) were compared for differences between percentages.

The study was approved by the Ethics Committee of the Faculty of Health Sciences of the University of the Free State. 


\section{Results}

Images from 309 patients were assessed for the study. The group included $191(61.8 \%)$ white and 118 (38.2\%) black patients. One hundred and seventy-six (57\%) patients were female and $133(43 \%)$ male, with ages ranging from $18-83$ years.

A spectrum of DS endings was seen extending from the lower third of L5 to the lower third of S3. The distribution frequency of levels of termination of the DS in all patients on MR images demonstrated that the median ending of the DS was located at the middle third of S2, as observed in 75 (24.3\%) patients (Fig. 2). In $15.2 \%$ of patients, the DS terminated at levels above the S1-S2 intervertebral disc space, while 13.9\% had DS levels below the lower border of S2 (Fig. 2). The caudal DS tip was located between the level of the S1-S2 interspace and the lower third of S2 in $70.9 \%$ of the study population.

Regarding racial distribution, the study group consisted of 118 (38.2\%) black and 191 (61.8\%) white patients. The mean DS tip levels of the black and white patients ( $22.9 \%$ and $25.1 \%$, respectively) were at the middle third of S2 (Table I). No statistical differences in the mean level of the DS tip were observed between black and white patients.

The study group was divided into the following age groups; the number of patients in each group is in brackets: 18 - 29 years (35), 30 - 39 years (63), 40 - 49 years (91), 50 - 59 years (64), 60 - 69 years (46) and 70 - 83 years (10). This was done to determine any notable difference in the position of the DS tip with increasing age. The mean DS levels in the age groups 18 - 29, $40-49,50-59$ and $70-83$ years were at the middle third of S2, while patients in the age groups $30-39$ and $60-69$ years showed a mean level in the upper third of S2. No statistically significant differences in DS levels were seen with increasing age.

A comparison of male and female patients was also performed to determine any gender-related differences in the caudal vertebral position of the DS tip. Results are shown in Table II. The mean levels for the male (25.6\%) and female (23.3\%) patients were both at the middle third of S2, with no statistical differences noted between the two genders.

\section{Discussion}

The supposition that the lower border of the DS tip was situated at the inferior edge of S2 had been widely accepted for many years, based mainly on published findings from autopsies performed on adults. ${ }^{11,12}$

Larsen et al. ${ }^{13}$ performed a radio-anatomical study of the DS in patients subjected to myelography. They reported that the tip of the DS ended at the S1-S2 intervertebral disc space in the majority of cases, and further described a range of DS tip endings extending from the L5-S1 to S4-S5 intervertebral disc spaces. Previous studies of the lumbar spine utilising MR imaging to visualise the DS demonstrated a mean level of termination at the middle third of S2, ${ }^{5,6,14}$ similar to the level found in our study.

In contrast to our findings, Macdonald et al. ${ }^{5}$ found minor gender differences in the level of DS termination, with the level ranging from the upper third of S1 to the upper third of S4, which is slightly lower than the spectrum found in our study.

The findings reported here are of particular importance as far as caudal block, CSI and spine surgery are concerned. Routine setting of the radiation field with CSI is at the inferior border of the S2 vertebra.
This recommendation has largely been based on myelography and autopsy studies performed in adults. ${ }^{2,11,12}$ Some of the limitations imposed on cadaveric studies are based on a predominantly aged group of subjects and include unselected subjects plus time-dependent shrinkage of the specimens. In a living population, MRI appears to be a reasonable alternative for determining the level of the DS and anatomy of the lumbosacral region. The advantages of MRI are (i) it identifies possible disorders in live healthy subjects, (ii) the spinal column is in the supine position, and (iii) the age range is wider when a living population is investigated compared with cadaveric studies.

Our study demonstrated a spectrum of DS endings ranging from the lower third of L5 to the lower third of S3, similar to the published findings from previous studies. ${ }^{8,15}$ We determined that, across the various groups included in this study, the DS most commonly terminated at the middle third of the S2 vertebra, which is consistent with the findings of Macdonald et al., ${ }^{5}$ although it differs from previous studies where the DS termination was reported at the upper third of $S 2 .{ }^{8,15}$ Binokay et al. ${ }^{8}$ attributed this difference from other studies to racial differences and techniques used in the study groups. As with other studies, ${ }^{14-16}$ we were unable to demonstrate a statistical difference between the level at which the DS terminated in black or white patients, and could find no association between age and the level of DS termination. We were also in agreement with others ${ }^{3,15}$ who could find no statistical difference between male and female patients. The study by Macdonald et al..$^{5}$ did, however, report a difference in the DS termination level between males and females.

The findings reported here are also of great consequence for CSI. The entire neuroaxis should be covered by ensuring that the DS is included in the spinal field. ${ }^{15,17}$ At our oncology centre, the routine placement of the CSI field is at the lower border of S2. According to our results, $13.9 \%$ of patients showed a DS termination below the lower border of S2, which is consistent with data published by Nazmy et al. ${ }^{16}$ who determined that the inferior border of the DS was lower than the inferior border of S2 in 31\% of patients. Dunbar et al. ${ }^{17}$ found that $14.7 \%$ of patients showed a DS termination below the S2-S3 junction, while Scharf et al ${ }^{15}$ reported similar findings, with $8.7 \%$ of patients having a DS lower than the S2-S3 junction. Consequently, these patients would be undertreated if the lower border of the CSI spinal field were placed at the inferior border of S2. This may lead to considerable geographical miss, probably with negative effects on local control of the disease

In $15.2 \%$ of the patients included in our investigation, the inferior border of the DS was situated above the S1-S2 intervertebral space. Dunbar et al. ${ }^{17}$ showed that in $16.6 \%$ of patients, the inferior border of the DS was at mid-S1, whereas data reported by Scharf et al. ${ }^{15}$ showed that $17.4 \%$ of patients had a DS termination at this level. On the basis of the above information, these patients would therefore receive unnecessary irradiation to critical structures if the lower border of the CSI spinal field was placed at the lower border of S2. Although the extent of this difference is minor, its effects - especially on the dose to the ovaries in female patients - would be considerable. The wide variation in the level of the inferior border of the DS and the failure to find any other tool to indicate its level accurately, make individualised treatment using MRI data of the spine a crucial element in the planning process. 


\section{ORIGINAL ARTICLE}

Our findings are also relevant to the performance of safe caudal block procedures, which are used for intra- and postoperative analgesia and the management of chronic pain. Crighton et al. ${ }^{6}$ suggested that,to increase the chances of performing a successful caudal block with minimal risk of dural puncture, the most frequent termination level of the DS should be known. They found a mean termination level of the DS at the middle third of S2 which compares favourably with our study. In the former study, the authors reported a mean distance between the sacrococcygeal membrane (SCM) and the DS of $60.5 \mathrm{~mm}$, with a range of $34-80 \mathrm{~mm}$. The distance of $34 \mathrm{~mm}$ is of substantial importance in clinical practice because the use of a 21 gauge $40 \mathrm{~mm}$ 'green' needle to perform a caudal block has the additional risk of puncturing the dura in some patients.

The results of the present study failed to demonstrate a difference in the dural sac termination level found in the South African population in comparison with the levels reported in various international studies; nor is there a statistical difference between gender, race and age groups. We did, however, determine that the routine placement of the portal field at the lower border of S2 adequately treats the majority of CSI patients. Nevertheless, we propose using spinal MRI to set the lower border of the CSI spinal field as this will benefit the patient in ensuring adequate coverage of the entire neuroaxis as well as minimising late gonadal toxicity. As MRI investigations are usually part of the workup for medulloblastoma and other neuroaxis seeding brain tumours, no additional costs need be incurred. Radiologists can and should make a note of the DS termination during these examinations to set a level for possible CSI spinal field.

\section{Acknowledgements}

We thank Professor Gina Joubert of the Department of Biostatistics, Faculty of Health Sciences, UFS, for assisting with the statistical analysis, and Daleen
Struwig (medical writer, Faculty of Health Sciences, UFS) for technical and editorial preparation of the manuscript.

1. Moore KL. Clinically Orientated Anatomy. 2nd ed. Baltimore: Williams \& Wilkins, 1985:606-618.

2. Berry M, Bannister LH, Standring SM. Gray's Anatomy. 38th ed. New York: Churchill Livingstone, 1995: 1212 .

3. Hansasuta A, Tubbs RS, Oakes WJ. Filum terminale fusion and dural sac termination: study in 27 cadavers. Pediatr Neurosurg 1999;30:176-179.

4. Lanier VS, McKnight HE, TrotterM. Caudal analgesia: an experimental and anatomical study. Am J Obstet Gynecol 1944;47:633-641

5. Macdonald A, Chatrath P, Spector T, Ellis H. Level of termination of the spinal cord and the dural sac: a magnetic resonance study. Clin Anat 1999;12:149-152.

6. Crighton IM, Barry BP, Hobbs GJ. A study of the anatomy of the caudal space using magnetic resonance imaging. Br J Anaesth 1997;78:391-395.

7. Ruggieri PM. A practical approach to magnetic resonance physics in spinal imaging. In: Modic MT, Masaryk TJ, eds. Magnetic Resonance of the Spine. 2nd ed. Philadelphia: Mosby, 1994:1-35.

8. Binokay F, Akgul E, Bicakci K, Soyupak S, Aksungur E, Sertdemir Y. Determining the level of the dural sac tip: magnetic resonance imaging in an adult population. Acta Radiol 2006; 47:397-400.

9. Phongkitkarum S, Jaovisidha S, Dhanachai M. Determination of the thecal sac ending using magnetic resonance imaging: clinical applications in craniospinal irradiation. J Med Assoc Thai 2004;87:1368-1373.

10. Saifuddin A, Burnett SJ, White J. The variation of position of the conus medullaris in an adult population. A magnetic resonance imaging study. Spine 1998; 23:1452-1456.

11. McCotter R. Regarding the length and extent of the human medulla spinalis. Anat Rec 1915;10:559-574.

12. Needles J. The caudal levels of termination of the spinal cord in American whites and American negroes. Anat Rec 1935;63:417-424.

13. Larsen JL, Olsen KO. Radiographic anatomy of the distal dural sac. A myelographic investigation of dimensions and termination. Acta Radiol 1991;32:214-219.

14. Kim JT, Bahk JH, Sung J. Influence of age and sex on the position of the conus medullaris and Tuffier's line in adults. Anaesthesiol 2003;99:1359-1363.

15. Scharf CB, Paulino AC, Goldberg KN. Determination of the inferior border of the thecal sac using magnetic resonance imaging: implications on radiation therapy treatment planning. Int J Radiat Oncol Biol Phys 1998;41:621-624.

16. Nazmy MS, Attalla EM, Refeat A. Effect of magnetic resonance myelography on the target volume in craniospinal irradiation in children. Clin Oncol 2009;21:14-18.

17. Dunbar SF, Barnes PD, Tarbell NJ. Radiologic determination of the caudal border of the spinal field in cranial spinal irradiation. Int J Radiat Oncol Biol Phys 1993;26:669-673.

\section{Fellowship announcement}

The following newly qualified radiologists and registrars have been elected for subspecialty fellowships.

From the University of Pretoria: Dr Scott Davies passed FC Rad (Diag) in2009. Taking up a neuroradiology fellowship at Royal Perth Hospital/Sir Charles Gairdner Hospital, Australia, at the end of 2010.

From the University of the Witwatersrand's Radiology Department: Dr Hanief Moosa, registrar, has been awarded the first ESOR visiting MSK scholarship for South Africa for 2010. He will spend 3 months at the University Hospital of Strasbourg, France, from September - November 2010.

From the University of KwaZulu-Natal, Greys Hospital, Pietermaritzburg: Dr Ruan Visagie passed FC Rad (Diag) in May 2010. He started a fellowship in musculoskeletal imaging on 1 July 2010 at McMaster University, Hamilton, Ontario, Canada. Dr Miranda Durand passed FC Rad (Diag) in October 2009 and started a fellowship in cardiac imaging on 1 July 2010 at the University of Toronto, Canada.

From the University of Cape Town/Groote Schuur Hospital: Dr Nuraan Abdurahman passed FC Rad (Diag) SA in 2009 and starts a fellowship in paediatric imaging in Melbourne, Australia, in July 2011.

From Tygerberg Hospital/ University of Stellenbosch: Dr Dirk van der Merwe passed FC Rad (Diag) in May 2010. Starts an MSK fellowship at the University of Calgary, Canada, in October 2010. Dr Arthur Maydell, registrar, starts a neuroradiology fellowship at Rush University, Chicago, in July 2011. Dr Pieter Janse van Rensburg passed MMed (Rad D) in 2009 and FRCR in 2010, starts a 2-year neuroradiology fellowship at the University of Utah, Salt Lake City, USA, on 1 July 2011.

The RSSA congratulates them - and hopes to see them all back in South Africa. 